implication in general) to the rule of syllogism-is wholly fallacious; the real source of the difference lies in the change of sense of the middle term; that to confound in a middle term the sensus compositi and the sensus divisi is a source of danger has been a commonplace of logic since the time of the scholastics. That an inept symbolism is made use of in mathematics, which has for a fundamental interest the point and the "variable" (i. e., individuals, or singulars) would be of no consequence, but Russell and Peano treat this "addition" as constituting an important improvement over the logic which preceded them-that of Peirce and his school-instead of which it is simply erroneous.

F. N. Cole, Secretary.

\title{
ON THE HEINE-BOREL PROPERTY IN THE THEORY OF ABSTRACT SETS.
}

BY DR. E. W. CHITTENDEN.

(Read before the American Mathematical Society, October 26, 1918.)

O. Veblen and N. J. Lennes have shown that in the presence of certain linear order axioms the Heine-Borel property is equivalent to the Dedekind cut axiom.* O. Veblent and R. L. Mooreł have used this property in systems of axioms for geometry and analysis situs.

M. Fréchet established the theorem that in a class (V) normale a subclass $\mathfrak{Q}$ has the Heine-Borel property if and only if $\mathfrak{Q}$ is compact and closed. $\S$ This result was extended to systems $\left(\mathfrak{Q} ; \mathrm{K}^{1367}\right)$ by $\mathrm{T}$. H. Hildebrandt.||

E. R. Hedrick calls attention to the fact that the Heine-Borel theorem, in the enumerable case, is a consequence of the closure of derived classes. T

* Cf. O. Veblen, "The Heine-Borel theorem," this Bulletin, vol. 10 (1904), p. 436-439.

$\dagger$ "A system of axioms for geometry, "Transactions Amer. Math. Society, vol. 5 (1904), pp. 343-384.

$\ddagger$ "On the foundations of plane analysis situs," ibid., vol. 17 (1916), p. 131 .

$\S$ Sur quelques points du calcul fonctionnel," Rendiconti di Palermo, vol. 22 (1906), p. 26.

|| "A contribution to the foundations of Fréchet's calcul fonctionnel," Amer. Journal of Math., vol. 34 (1912), pp. 281-282.

T "On properties of a domain for which the derived set of any set is closed," Transactions Amer. Math. Society, vol. 12 (1911), pp. 285-294. 
In a previous paper* I have shown that the Heine-Borel property implies self-compactness and extremality in any set $\mathcal{L}$ of Fréchet. The present paper considers the Heine-Borel property in relation to the closure of derived classes and the property of separability. The converse of the theorem of Hedrick is established.

1. Let $\Re$ denote a fundamental set of elements called points such that for each point $P$ and subset $\mathfrak{B}$ it is determined whether or not $P$ is a limiting point of $\mathfrak{B}$. F. Riesz $\dagger$ regards the following four axioms as fundamental in the theory of systems $\Re$ :

$(A)$ If a point $P$ is a limiting point of a set $\mathfrak{P}$ and $\mathfrak{B}$ is a subset of $\mathfrak{Q}$, then $P$ is a limiting point of $\mathfrak{Q}$.

(B) If $\mathfrak{P}=\mathfrak{P}_{1}+\mathfrak{P}_{2}$ and $P$ is a limiting point of $\mathfrak{P}$, then $P$ is a limiting point of $\mathfrak{P}_{1}$ or $\mathfrak{P}_{2}$.

(C) If $P$ is a limiting point of $\mathfrak{B}$, then $\mathfrak{P}$ contains at least two elements. $\ddagger$

$(D)$ A point $P$ is determined uniquely by the sets of which it is a limiting point.

A point $P$ is interior to a set $\mathfrak{S}$ if $\mathfrak{S}$ contains $P$ and a point of every set which has $P$ for a limiting point. $\$$

A family $(\mathfrak{S}$ ) of sets $\mathfrak{S}$ of points covers a set $\mathfrak{Q}$ if every point of $\mathfrak{Q}$ is interior to some class $\mathfrak{S}$.

A class $\mathfrak{Q}$ has the Heine-Borel property if every family ( $\mathfrak{Y}$ ) which covers $\mathfrak{Q}$ contains a finite subfamily

$$
\mathfrak{S}_{1}, \mathfrak{S}_{2}, \mathfrak{S}_{3}, \cdots, \mathfrak{S}_{n} \text {, }
$$

which also covers $\mathfrak{Q}$. The enumerable case of this property is defined by the restriction "if every enumerable family ( $\mathfrak{S})$, etc." When the families $(\mathfrak{S})$ are not subject to this limitation I shall speak of the general case.

Theorem 1. If $\Re$ satifies axiom $(A)$ and $\mathfrak{P}$ is a subset of $\Re$ possessing the Heine-Borel property, then any closed subset of $\mathfrak{P}$ has the same property.

\footnotetext{
* "On the converse of the Heine-Borel theorem in a Riesz domain," this BulletiN, vol. 21 (1915), pp. 179-183. Cf. Theorems I and IV.

† Cf. "Stetigkeitsbegriff und abstrakte Mengenlehre," Atti del IV Congresso Inter. dei Mat., Roma (1908), vol. 2, pp. 18-24.

$\ddagger$ From axioms $(B)(C)$ it follows that, if $\mathfrak{P}$ has a limiting point, $\mathfrak{P}$ is an infinite class.

\$In the previous paper I assumed that this point be distinct from $P$. R. L. Root called my attention to the fact that this condition is unnecessary in view of axioms $(B)$ and $(C)$. The removal of the restriction permits a generalization of Theorem I of the previous paper, also suggested by Root.
} 
Let $\mathfrak{Q}$ be a closed subset of $\mathfrak{B}$ and $(\mathfrak{S})$ be a family of sets covering $\mathfrak{\Omega}$. Denote by $\subseteq$ the class of all points of $\Re$ not contained in $\mathfrak{Q}$. From the closure of $\mathfrak{Q}$ and axiom $(A)$ it follows that no subset of $\mathfrak{Q}$ has a limiting point in $\subseteq$. The family of sets obtained by adding $\subseteq$ to $(\mathfrak{S})$ covers $\mathfrak{B}$, and must contain a finite subfamily with the same property. This finite subfamily contains $\mathfrak{S}$, but $\subseteq$ contains no point of $\mathfrak{O}$. Hence $(\mathfrak{S})$ contains a finite subfamily which covers $\mathfrak{Q}$, as was to be proved.

The following theorem is a generalization of theorem 1 of the previous paper, suggested to me by R. E. Root. As the proof of the present theorem is only slightly different from the proof of the original theorem, it is omitted here.

THEOREm 2. If $\Re$ satisfies axiom $(A)$ and $\mathfrak{B}$ is any subset of $\Re$ possessing the Heine-Borel property in the enumerable (or general case), then $\mathfrak{B}$ is self-compact; that is, every infinite subclass of $\mathfrak{P}$ has a limiting point in $\mathfrak{P}$.

Theorems 1 and 2 are easily seen to hold for the general sets (B) recently introduced by Fréchet.*

2. It follows from theorem 2 that if $\Re$ satisfies axiom $(A)$ and contains an infinite class with the Heine-Borel property then there is an element in $\Re$ which is a limiting point of an enumerable set. The following example shows that not all points of $\Re$ need have this property. Let $\Re$ denote the closed interval $(0,1)$ with the additional point $P=2$. Limiting point is defined as usual for the interval $(0,1)$. The point $P=2$, however, is to be a limiting point of every subset of $\Re$ which has the power of the continuum. Then $\Re$ satisfies axioms $(A),(B),(C),(D)$, and also has the Heine-Borel property. Theorem 2 is valid, but the point $P=2$ is not a limiting point of any enumerable set. The derived class of the rational points of $\Re$ is the interval $(0,1)$, which is not closed in $\Re$. Hence closure of derived classes is not implied by the Heine-Borel property in the set $\Re$ as conditioned by Riesz. If we assume in place of axiom $(D)$ the stronger axiom:

$\left(D^{\prime}\right)$ If $P$ is a limiting point of a set $\mathfrak{B}$, then $\mathfrak{P}$ contains an enumerable set $\mathfrak{S}$, of which $P$ is the only limiting point; we shall obtain a system $\Re$ in which closure of derived classes is implied by the Heine-Borel property. We have

Theorem 3. If $\Re$ satisfies axioms $(A),(B),(C),\left(D^{\prime}\right)$ and any class $\mathfrak{\Omega}$ in $\Re$ possesses the Heine-Borel property in the enumerable case, the derived class of every subclass of $\mathfrak{Q}$ is closed.

*Comptes Rendus, vol. 165 (1917), pp. 359-60. 
We will first prove that $\mathfrak{Q}$ is closed. From axiom $\left(D^{\prime}\right)$ any limiting point $Q$ of $\Re$ is a unique limiting point of some enumerable subset $\subseteq$ of $\mathfrak{D}$. $\subseteq$ is an infinite set (axioms $(A),(B)$, $(C))$ and from theorem 2 has a limiting point in $\subseteq$. We shall now show that a contradiction results if we assume that $\mathfrak{D}$ contains a set whose derived set is not closed. Let $\mathfrak{D}$ be a subset of $\mathfrak{Q}$ such that $\mathfrak{D}^{\prime}$ has a limiting point $Q$ not in $\mathfrak{D}^{\prime}$. Then there is a sequence of points of $\mathfrak{Q}^{\prime}$,

$$
\mathfrak{D}_{1}, \mathfrak{D}_{2}, \mathfrak{D}_{3}, \cdots
$$

with the unique limiting point $Q$. Denote by $\mathfrak{S}_{n}$ the class of all points of $\Re$ excepting the points $\mathfrak{S}_{n+h}(h=1,2,3, \cdots)$, and $\mathfrak{Q}$. It is evident that each point of $\mathfrak{Q}$ is interior to at least one of the classes $\mathfrak{S}_{n}$. Denote by $\mathfrak{S}$ the set of all points not contained in the set $\mathfrak{O}$. Then the point $Q$ is interior to $S$. The family

$$
\Im, \mathfrak{S}_{1}, \mathfrak{S}_{2}, \cdots
$$

is enumerable and covers the set $\mathfrak{Q}$. It contains a finite subfamily which also covers $\mathfrak{Q}$ and of which $\subseteq$ is a member. No class $\mathfrak{S}_{n}$ contains more than $n$ of the points $O_{n}$. Therefore some point $O_{n_{0}}$ must be interior to $\subseteq$. Since the point $O_{n_{0}}$ is an element of the set $\mathfrak{D}^{\prime}$, some point of $\mathfrak{D}$ must belong to $\mathfrak{S}$. This is the desired contradiction.

In a class $\&$ of Fréchet* a point $P$ is a limiting point of a class $\mathfrak{B}$ if and only if $\mathfrak{P}$ contains a sequence of distinct elements with the limit $P$. The class $\&$ is a class $\Re$ satisfying axioms $(A),(B),(C),\left(D^{\prime}\right)$. From theorem 3 and a theorem of E. R. Hedrick $\uparrow$ we have at once:

Theorem 4. In any set $\mathfrak{R}$ of Fréchet a necessary and suffcient condition that a subset $\mathfrak{B}$ of $\mathbb{Q}$ possess the Heine-Borel property is that $\mathfrak{B}$ be compact, closed, and that the derived set of every subset of $\mathfrak{B}$ be closed.

3. F. Hausdorff $\ddagger$ employs a form of the Heine-Borel property which does not imply the closure of derived sets in every system $\mathbb{R}$ of Fréchet. The Hausdorff form of this property may be defined as follows: If a class $\mathfrak{B}$ is covered by a family (\$) of regions\$ (\$) then $\mathfrak{B}$ is covered by a finite subset of $(\$)$.

* Rendiconti di Palermo, loc. cit., p. 1, 2.

$\dagger$ Loc. it., p. 286.

‡ Grundzüge der Mengenlehre, Veit and Co., Leipzig (1914), p. 231.

$\$$ A region (Gebiet) is a set whose every element is an interior element. Cf. Hausdorff, loc. cit., p. 215, footnote 1 . 
Let the class $\mathfrak{R}$ be the interval $(0,1)$. We define limit as usual in $(0,1)$ with the single exception of the sequence of points $x=1 / m(m=2,3, \ldots)$. The sequence $\Sigma(1 / m)$ is to have the unique limit $x=1$. Any subset $\mathfrak{P}$ of $\mathfrak{R}$ is compact, but derived classes are not closed. Let $\mathfrak{O}$ denote the irrational points of the interval $(0,1 / 2)$. Then $\mathfrak{D}^{\prime}$ is the interval $(0,1 / 2)$, while $\mathfrak{D}^{\prime \prime}$ contains $x=1$. Nevertheless it is easy to see that any closed subset of $\mathbb{R}$ has the Hausdorff form of the Heine-Borel property. We give an example of a family $(\mathfrak{F})$ which covers $\mathbb{R}$ and cannot be replaced by a finite subfamily.

Let $\mathfrak{S}_{n}$ be the class of all points of $(0,1)$ except the points $x=1$ and $x=1 / m(m>n)$. Denote by $\mathfrak{S}_{0}$ the class of all points not an irrational point of the interval $(0,1 / 2)$. Then

$$
\mathfrak{S}_{0}, \mathfrak{S}_{1}, \ldots, \mathfrak{S}_{n}, \ldots
$$

is an enumerable family which covers $\mathfrak{B}$. No class $\mathfrak{S}_{n}$ contains more than a finite number of the points $x=1 / m$, and no point $x=1 / m(m>1)$ is interior to $\mathfrak{S}_{0}$. It follows that the family $(\mathfrak{S})$ cannot be replaced by a finite subfamily. The set $\mathfrak{S}_{0}$ is not a region.

4. Fréchet* has studied classes (B) in which for each element there is given a family ( $\mathfrak{B})$ of neighborhoods (voisinages). We shall impose the following conditions on the families ( $\mathfrak{B})$ : (1) For every element (point) $P$ the corresponding family $(\mathfrak{B})$ is enumerable and is denoted by $\left(\mathfrak{B}^{m}\right) ;(2)$ the point $P$ is contained in every neighborhood $\mathfrak{B}^{m}$ of $P$ and is the only such point: (3) in a family $\left(\mathfrak{B}^{m}\right), \mathfrak{B}^{m}$ contains $\mathfrak{B}^{m+1} ;(4)$ if a point $Q$ is in a neighborhood $\mathfrak{B}^{m}$ of $P$ then $P$ is in the corresponding neighborhood $\mathfrak{B}^{m}$ of $Q . \dagger$

A point $P$ is a limiting point of a class $\mathfrak{B}$ if every neighborhood of $P$ contains a point of $\mathfrak{B}$ distinct from $P$.

Theorem 5. If $\mathfrak{Q}$ is a set in a system ( $\mathfrak{B})$ with the HeineBorel property, and if $\mathfrak{Q}^{\prime}$ contains $\mathfrak{Q}$, then $\mathfrak{Q}$ is compact, perfect, and separable.

Since $\mathfrak{Q}^{\prime}$ contains $\mathfrak{Q}$ and $\mathfrak{Q}$ is compact (theorem 2) it follows that $\mathfrak{Q}$ is perfect. We have to prove that $\mathfrak{Q}$ contains an enumerable subset $\mathbb{E}$ such that $\mathfrak{Q}=\&^{\prime}$.

Each point $P$ is interior to the corresponding neighborhood

* Comptes Rendus, loc. cit.

$\uparrow$ The class (B) admits a definition of a symmetric distance function $\delta$, such that $\delta(P, Q)=0$ if and only if $P=Q$. 
$\mathfrak{B}^{m}$. The system of neighborhoods $\mathfrak{B}^{m}$ for fixed $m$ covers $\mathfrak{Q}$ and may be replaced by a finite subsystem,

$$
\mathfrak{B}^{m 1}, \quad \mathfrak{B}^{m 2}, \ldots \mathfrak{B}^{m k} ;
$$

such that each point of $\mathfrak{Q}$ is interior to some class $\mathfrak{B}^{m k}$ and each class $\mathfrak{B}^{m k}$ is a neighborhood of a point $Q^{m k}$ of the class $\mathfrak{O}$. Let $\&$ be the class of all points $Q^{m k}$. Since every point $P$ of $\mathfrak{Q}$ is interior to some set $\mathfrak{B}^{m k}$, it follows from condition (4) that $Q^{m k}$ is contained in the neighborhood $\mathfrak{B}^{m}$ of $P$. Therefore $P$ is a limiting point of the class $E$.

UNIVERSITY OF Iowa.

This article was in type before the writer learned of the existence of an article by Fréchet (Bulletin de la Societé mathématique de France, volume 35, 1917), in which it is shown that the closure of derived classes is a consequence of the Heine-Borel property in the case of a general system ? Theorem 3 of the present paper is a generalization of this result.

E. W. Chittenden.

\section{INTEGRALS AROUND GENERAL BOUNDARIES.}

BY PROFESSOR P. J. DANIELL.

THE concept of a boundary integral has been extended to curves of the type $x=x(t), y=y(t)$, where $x(t), y(t)$ are absolutely continuous functions of a parameter $t$. In this case the curves are more or less simple and have tangents "nearly everywhere." In applications to physics however the boundary must be considered rather as a boundary of a set (in the sense of the theory of point sets). The boundary will be, in general, a collection of points without definite tangents at all. This paper sets out a method by which such boundary integrals can be defined under certain restrictions placed on the two integrand functions $u, v$. The method depends on the concept of absolutely additive functions of sets.* The writer believes that these restrictions could be lightened and that there is a wide field here for further investigation.

*J. Radon, Wiener Sitzungsberichte, vol. 122 (1913), p. 1295. W. H. Ycung, Proceedings London Math. Society, vol. 13 (1914), p. 109. 